\title{
Rapid Deorphanization of Human Olfactory Receptors in Yeast
}

Emily A. Yasi ${ }^{1}$, Sara L. Eisen ${ }^{1}$, Hanfei Wang ${ }^{1}$, Widianti Sugianto ${ }^{1}$, Anita R. Minniefield ${ }^{1}$, Kaitlyn A. Hoover ${ }^{1}$, Paul J. Branham ${ }^{1}$, Pamela Peralta-Yahya ${ }^{1,2^{*}}$

\section{Table of Contents}

Materials and Methods

Page

Table S1. Sequence identity of olfactory receptors expressed in the colon

Table S2. Table of primers

$\mathrm{S} 2$

Table S3. Table of plasmids

Table S4. Table of strains

Table S5. Table of chemicals in the chemical panel

Table S6. Abbreviations and chemical descriptors used in the principle component analysis

Table S7. Chemical space coordinates of the screened chemicals

Table S8. $R^{2}$ values for dose response fits. $\quad S 7$

Figure S1. Dose response curve of OR2W3 with nerol $\quad$ S8

Figure S2. Dose response curves of colon ORs with chemical hits (false positives). $\quad$ S8

Figure S3. Dose response curves of OR2L13 and OR51B5 with validated chemicals S9

Figure S4. Chemical toxicity to yeast-based OR sensor

Figure S5. Changes in olfactory receptor gene expression levels in the presence of chemicals

Figure S6. Dose response curves of OR2A7 and OR2W3 with farnesol. S10

Figure S7. Eigenvalues for principle component determination 
Materials. Tert-butylbenzene (B90602) and 3-phenylbutyraldehyde (289027) were purchased from Sigma-Aldrich. Nonanoic acid (N0288) and nerol (N0077) were purchased from TCl Chemicals. Supplier information for the chemical panels can be found in Table S5.

Chemical toxicity to yeast-based OR sensor. Overnight cultures of PPY1802, PPY1804, PPY1806, and PPY1807 where diluted to an $\mathrm{OD}_{600}=1$ in $5 \mathrm{~mL}$ of $\mathrm{SD}\left(\mathrm{HL}^{-}\right)$. Cultures were incubated for 4 hours at $30^{\circ} \mathrm{C}, 250 \mathrm{rpm}$ with varying chemical concentrations with a final DMSO concentration of $1 \% . \mathrm{OD}_{600}$ was measured after incubation. The experiments were run in triplicate.

mRNA Quantification. Following the OR biosensing protocol, between $2 \times 10^{7}$ and $5 \times 10^{7}$ cells were pelleted and total RNA was extracted using Qiagen RNeasy Mini Kit. RNA concentration was measured using a Nanodrop Lite spectrophotometer and stored at $-80^{\circ} \mathrm{C}$. Reverse transcription was executed using $700 \mu \mathrm{g}$ of total RNA using the QuantiTect Reverse Transcription kit (Qiagen). Quantitative PCR reactions were set up using the QuantiTect SYBR Green PCR kit (Qiagen) using cDNA from $42 \mathrm{ng}$ of mRNA and read using a StepOnePlus Real-Time PCR system (Applied Biosciences). Reactions were set up as technical triplicates using primers EY270/EY271 for OR10S1, EY272/EY273 for OR2A7, EY274/EY275 for OR2T4, and ACT-F/ACT-R for actin. OR expression were normalized to the housekeeping gene, ACT1, that encodes actin and relative expression of chemical versus no chemical (DMSO) was compared using the comparative $\mathrm{C}_{\mathrm{T}}$ method (Livek and Schmittgen, 2001) using the equations below.

$$
\begin{gathered}
\Delta C_{T_{-} \text {Sample }}=\text { avg } C_{T_{-} \text {sample }}-\text { avg } C_{T_{-} \text {Actin }} \\
\Delta \Delta C_{T}=\Delta C_{T}-\Delta C_{T_{-} D M S O} \\
2^{-\Delta \Delta C_{T}=\text { Mean relative expression of gene of interest compared to DMSO control }} \\
2^{-\left(\Delta \Delta C_{T} \pm \text { StDev }\right)}=\text { Range of fold change in expression relative to DMSO control } \\
\text { StDev }=\text { standard deviation of } \Delta C_{T_{-} \text {Sample }}
\end{gathered}
$$

Table S1. Sequence identity of ectopically expressed olfactory receptors in the colon

\begin{tabular}{|l|l|l|l|l|l|l|l|}
\hline & OR2A7 & OR2T4 & OR2A42 & OR2W3 & OR51B5 & OR2L13 & OR10S1 \\
\hline OR2A7 & & & & & & & \\
\hline OR2T4 & $39.90 \%$ & & & & & & \\
\hline OR2A42 & $71.30 \%$ & $44.00 \%$ & & & & & \\
\hline OR2W3 & $42.70 \%$ & $41.60 \%$ & $46.90 \%$ & & & & \\
\hline OR51B5 & $31.80 \%$ & $28.80 \%$ & $32.20 \%$ & $27.60 \%$ & & & \\
\hline OR2L13 & $39.50 \%$ & $49.20 \%$ & $42.20 \%$ & $39.30 \%$ & $26.20 \%$ & & \\
\hline OR10S1 & $39.60 \%$ & $36.40 \%$ & $41.70 \%$ & $41.60 \%$ & $28.20 \%$ & $40.30 \%$ & \\
\hline
\end{tabular}

Table S2. Table of primers

\begin{tabular}{|l|l|}
\hline Name & Sequence \\
\hline EY46 & ACTTCTTGCTCATTAGAAAG \\
\hline HW12 & CTTTTCGGTTAGAGCGGATC \\
\hline EY270 & CTGTCAGCCGTTACATTATCC \\
\hline
\end{tabular}




\begin{tabular}{|l|l|}
\hline EY271 & CCACAATACAGCAGACGAAAG \\
\hline EY272 & CCGTGAAGTCAGCGTAAG \\
\hline EY273 & TTCAGCATCGGGTTAAACAG \\
\hline EY274 & TTCAGACAGAGCAAGCACC \\
\hline EY275 & ACGAAAATGACGACACACAG \\
\hline ACT-F & TTCTGAGGTTGCTGCTTTGG \\
\hline ACT-R & ACCGACGATAGATGGGAAGAC \\
\hline HW1 & GCAATCTAATCTAAGTTTTAATACAAAGGATCC \\
\hline HW4 & AGAATGAGATTCCTTGCCC \\
\hline HW7 & TTCCTTGCCTAGTTCTCTCTTACC \\
\hline HW8 & TTCCATTGCCTTTTGGAAGGC \\
\hline HW9 & GGTACCAATTCTATGGGTGGTG \\
\hline PB89 & TTCCTTCAGGAAGCTGAAGATACC \\
\hline PB116 & CGGCGGCGGGCTA \\
\hline PB118 & CAGTGCACGTTCAACACCC \\
\hline HW15 & AGGAGGGCTTTGGGCAAGGAATCTCATTCTGTGAGCAAGGGCGAGG \\
\hline HW18 & TTGTTGGGTAAGAGAGAACTAGGCAAGGAAGTGAGCAAGGGCGAGG \\
\hline HW19 & GTTGAACCAGCCTTCCAAAAGGCATGGAAGTGAGCAAGGGCGAGG \\
\hline HW20 & CATTTGTTCACCACCCATAGAATTGGTACCGTGAGCAAGGGCGAGG \\
\hline PB88 & GTGTTCGGTATCTTCAGCTTCCTGAAGGAAGTGAGCAAGGGCGAG \\
\hline PB115 & AGCACCGCAGGTAGCCCGCCGCCGGTGAGCAAGGGCGAGG \\
\hline PB117 & GTTCTGGGTGTTGAACGTGCACTGGTGAGCAAGGGCGAGG \\
\hline
\end{tabular}

Table S3. Table of Plasmids.

\begin{tabular}{|c|c|c|c|}
\hline Number & Name & Description & Source \\
\hline PPY111 & pKM111 & pESC-His3-P $P_{\mathrm{TEF} 1}-\mathrm{P}_{\mathrm{ADH} 1}$ & Mukherjee et al. (2015) \\
\hline PPY586 & pKM586 & pRS415- $P_{\mathrm{FlG} 1-\mathrm{eGFP}-L e u 2}$ & Mukherjee et al. (2015) \\
\hline PPY1658 & pHW3 & pESC-HIS3-P ${\text { TEF1 } 1-O R 2 A 42-P_{A D H 1}}$ & This study \\
\hline PPY1661 & pHW6 & pESC-HIS3-P TEF1-OR2W3- PADH1 $_{1}$ & This study \\
\hline PPY1663 & pHW7 & pESC-HIS3-P $\mathrm{TEF} 1-\mathrm{OR} 2 \mathrm{~T} 4-\mathrm{P}_{\mathrm{ADH} 1}$ & This study \\
\hline PPY1715 & pHW18 & pESC-HIS3-P & This study \\
\hline PPY1795 & pPB8 & 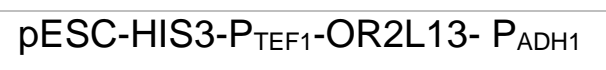 & This study \\
\hline PPY1717 & pHW2O & pESC-HIS3-P TEF1-OR10S1- $_{\text {ADH1 }}$ & This study \\
\hline PPY1718 & pHW21 & 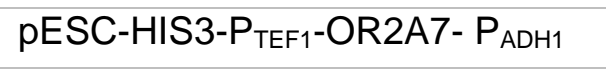 & This study \\
\hline PPY1719 & pHW22 & 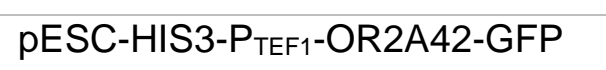 & This study \\
\hline PPY1720 & pHW23 & pESC-HIS3-P TEF1-OR51B5-GFP & This study \\
\hline
\end{tabular}




\begin{tabular}{|c|c|c|c|}
\hline PPY1734 & $\mathrm{pHW} 30$ & 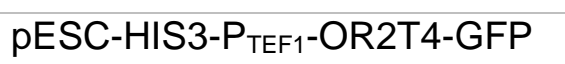 & This study \\
\hline PPY1737 & pHW33 & pESC-HIS3-P $\mathrm{PEF} 1_{\mathrm{T}}-\mathrm{OR} 2 \mathrm{~W} 3-\mathrm{GFP}$ & This study \\
\hline PPY1947 & pPB59 & pESC-HIS3-P TEF1 - OR2A7-GFP & This study \\
\hline PPY1948 & pPB60 & pESC-HIS3-P $\mathrm{TEF} 1-\mathrm{OR} 10 \mathrm{~S} 1-\mathrm{GFP}$ & This study \\
\hline PPY1930 & pPB54 & pESC-HIS3-P ${ }_{\mathrm{TEF} 1}-\mathrm{OR} 2 \mathrm{~L} 13-\mathrm{GFP}$ & This study \\
\hline
\end{tabular}

Table S4. Table of Strains.

\begin{tabular}{|c|c|c|}
\hline Strain Number & Description & Source \\
\hline PPY140 & $\begin{array}{l}\text { S. cerevisiae MATa ade2-1 ura3-1 his3-11 trp1-1 leu2-3 } \\
\text { leu2-112 can1-100 } \Delta \text { far1, } \Delta \text { sst2, } \Delta \text { ste2 }\end{array}$ & $\begin{array}{l}\text { Mukherjee et al. } \\
(2015)\end{array}$ \\
\hline PPY1800 & PPY140 carrying pKM111 and pKM586 & This study \\
\hline PPY1801 & PPY140 carrying pHW3 and pKM586 & This study \\
\hline PPY1802 & PPY140 carrying pHW6 and pKM586 & This study \\
\hline PPY1803 & PPY140 carrying pHW7 and pKM586 & This study \\
\hline PPY1804 & PPY140 carrying pHW18 and pKM586 & This study \\
\hline PPY1805 & PPY140 carrying pPB8 and pKM586 & This study \\
\hline PPY1806 & PPY140 carrying pHW20 and pKM586 & This study \\
\hline PPY1807 & PPY140 carrying pHW21 and pKM586 & This study \\
\hline PPY1949 & PPY140 carrying pHW22 & This study \\
\hline PPY1950 & PPY140 carrying pHW23 & This study \\
\hline PPY1951 & PPY140 carrying pHW30 & This study \\
\hline PPY1952 & PPY140 carrying pHW33 & This study \\
\hline PPY1953 & PPY140 carrying pPB59 & This study \\
\hline PPY1954 & PPY140 carrying pPB60 & This study \\
\hline PPY1955 & PPY140 carrying pPB54 & This study \\
\hline
\end{tabular}

Table S5. Table of chemicals in the chemical panel

\begin{tabular}{|l|c|c|c|}
\hline Chemical & CAS \# & Supplier & Catalog \# \\
\hline$(-)$-limonene & $5989-54-8$ & TCl Chemicals & L0132 \\
\hline (+)-carvone & $6485-40-1$ & TCl Chemicals & C0703 \\
\hline (+)-menthol & $2216-51-5$ & TCl Chemicals & M0826 \\
\hline 1,2,3-trimethylbenzene & $526-73-8$ & TCl Chemicals & T0468 \\
\hline 1-butanol & $71-36-3$ & TCl Chemicals & B0944 \\
\hline 1-butoxy-2-propanol & $5131-66-8$ & TCl Chemicals & B0864 \\
\hline 1-dodecanol & $112-53-8$ & Alfa Aesar & A12228 \\
\hline 1-octanol & $111-87-5$ & TCl Chemicals & O0036 \\
\hline 1-propanol & $71-23-8$ & TCl Chemicals & P0491 \\
\hline
\end{tabular}




\begin{tabular}{|c|c|c|c|}
\hline 2,3-heptadione & $96-04-8$ & TCl Chemicals & H0422 \\
\hline 2,5-dimethylpyrazine & $123-32-0$ & TCI Chemicals & D1526 \\
\hline 2-methyl-1-butanol & $137-32-6$ & TCl Chemicals & M0175 \\
\hline 3-hexanone & $589-38-8$ & TCI Chemicals & $\mathrm{H} 0115$ \\
\hline 3-pentanol & $584-02-1$ & TCI Chemicals & P0057 \\
\hline acetic acid & $64-19-7$ & VWR & BDH3092 \\
\hline alpha-pinene & $80-56-8$ & Sigma-Aldrich & 305715 \\
\hline anisaldehyde & $123-11-5$ & TCl Chemicals & A1674 \\
\hline beta-pinene & $127-91-3$ & Sigma-Aldrich & CRM40433 \\
\hline butyric acid & $107-92-6$ & TCl Chemicals & B0754 \\
\hline coumarin & $91-64-5$ & TCl Chemicals & C0395 \\
\hline ethyl decanoate & $110-38-3$ & TCI Chemicals & D0022 \\
\hline ethyl laurate & $106-33-2$ & TCl Chemicals & L0013 \\
\hline ethyl myristate & $124-06-1$ & TCl Chemicals & M0479 \\
\hline ethyl octanoate & $106-32-1$ & TCl Chemicals & 00030 \\
\hline ethyl palmitate & $628-97-7$ & Alfa Aesar & A15694 \\
\hline ethyl stearate & $111-61-5$ & TCl Chemicals & S0079 \\
\hline eugenol & $97-53-0$ & Alfa Aesar & A14332 \\
\hline farnesol & $4602-84-0$ & TCI Chemicals & T0608 \\
\hline geraniol & $106-24-1$ & TCI Chemicals & G0027 \\
\hline heptanoic acid & $111-14-8$ & Alfa Aesar & A17704 \\
\hline hexanoic acid & $142-62-1$ & Alfa Aesar & A13789 \\
\hline isovaleric acid & $503-74-2$ & TCI Chemicals & M0182 \\
\hline lactic acid & $50-21-5$ & Alfa Aesar & 36415 \\
\hline lauric acid & $143-07-7$ & TCI Chemicals & L0011 \\
\hline lilial & $80-54-6$ & TCI Chemicals & B1145 \\
\hline linalool & $78-70-6$ & TCI Chemicals & L0048 \\
\hline lyral & $31906-04-4$ & Sigma-Aldrich & 95594 \\
\hline melatonin & $73-31-4$ & Sigma-Aldrich & M5250 \\
\hline methyl decanoate & $110-42-9$ & Alfa Aesar & A15658 \\
\hline methyl laurate & $111-82-0$ & Alfa Aesar & A12492 \\
\hline methyl myristate & $124-10-7$ & Alfa Aesar & A10257 \\
\hline methyl octanoate & $111-11-5$ & Alfa Aesar & A10991 \\
\hline methyl palmitate & $112-39-0$ & TCl Chemicals & S0311 \\
\hline methyl stearate & $112-61-8$ & TCl Chemicals & S0312 \\
\hline m-xylene & $108-38-3$ & Sigma-Aldrich & 95670 \\
\hline myristic acid & $544-63-8$ & TCI Chemicals & M0476 \\
\hline nonanal & $124-19-6$ & TCI Chemicals & N0296 \\
\hline octanal & $124-13-0$ & Alfa Aesar & A10901 \\
\hline
\end{tabular}




\begin{tabular}{|l|c|c|c|}
\hline octanoic acid & $124-07-2$ & Alfa Aesar & A11149 \\
\hline palmitic acid & $57-10-3$ & TCl Chemicals & P0002 \\
\hline p-cymene & $99-87-6$ & TCl Chemicals & C0513 \\
\hline pentadecanoic acid & $1002-84-2$ & Alfa Aesar & A14664 \\
\hline propionic acid & $79-09-4$ & TCl Chemicals & P05000 \\
\hline sotolon & $28664-35-9$ & TCl Chemicals & D1851 \\
\hline stearic acid & $57-11-4$ & TCl Chemicals & S0163 \\
\hline undecanal & $112-44-7$ & TCl Chemicals & U0009 \\
\hline vanillin & $121-33-5$ & Alfa Aesar & A11169 \\
\hline
\end{tabular}

Table S6. Abbreviations and Chemical Descriptors for PCA Analysis

\begin{tabular}{|c|c|}
\hline Descriptors & Abbreviations \\
\hline Molecular Weight & MW \\
\hline Carbon atom count & CAC \\
\hline Hydrogen atom count & HAC \\
\hline Oxygen atom count & OAC \\
\hline Nitrogen atom count & NAC \\
\hline Hydrogen-bond donors & HBD \\
\hline Hydrogen-bond acceptor & HBA \\
\hline Rotatable bonds & RB \\
\hline Stereocenter count & SC \\
\hline Topological polar surface area & TPSA \\
\hline Number of rings & NOR \\
\hline Aromatic ring count & ARC \\
\hline Ring system count & RSC \\
\hline Size of largest ring & SLR \\
\hline Van der Waals surface area & VSA \\
\hline Amine & - \\
\hline Alcohol & - \\
\hline Ether & - \\
\hline Aldehyde & - \\
\hline Ketone & - \\
\hline Carboxylic Acid & - \\
\hline Ester & - \\
\hline & \\
\hline
\end{tabular}


Table S7. Chemical space coordinates of the screened chemicals

\begin{tabular}{|c|c|c|c|c|c|}
\hline Chemical Space & $\begin{array}{c}\text { PC1 } \\
\mathbf{( 2 8 \%} \\
\text { variance) }\end{array}$ & $\begin{array}{c}\text { PC2 } \\
\mathbf{( 1 9 \%} \\
\text { variance) }\end{array}$ & $\begin{array}{c}\text { PC3 } \\
\mathbf{( 1 6 \%} \\
\text { variance) }\end{array}$ & Members & Population (\%) \\
\hline 1 & - & - & - & 9 & 15.8 \\
\hline 2 & + & - & - & 8 & 14.0 \\
\hline 3 & - & - & + & 6 & 10.5 \\
\hline 4 & + & - & + & 8 & 14.0 \\
\hline 5 & + & + & - & 2 & 3.5 \\
\hline 6 & - & + & - & 13 & 22.8 \\
\hline 7 & + & + & + & 6 & 10.5 \\
\hline 8 & - & + & + & 5 & 8.8 \\
\hline & & & Total: & 57 & 100 \\
\hline
\end{tabular}

Table S8. $R^{2}$ values for dose response fits

\begin{tabular}{|l|l|l|l|}
\hline Figure & OR & Chemical & $\mathbf{R}^{\mathbf{2}}$ \\
\hline 3A, 4A & OR2A7 & $\alpha$-pinene & 0.98946 \\
\hline 3A, 4B & OR2T4 & $\alpha$-pinene & 0.99269 \\
\hline 3B, 4C & OR2A7 & lilial & 0.97004 \\
\hline 3B, 4D & OR2T4 & lilial & 0.99854 \\
\hline 3B & OR10S1 & lilial & 0.99623 \\
\hline 3C & OR51B5 & farnesol & 0.99585 \\
\hline 3D & OR2T4 & undecanal & 0.90762 \\
\hline 3D & OR10S1 & nonanal & 0.99651 \\
\hline 4A & OR2A7 & ß-pinene & 0.99659 \\
\hline 4C & OR2A7 & tert-butylbenzene & 0.99853 \\
\hline
\end{tabular}




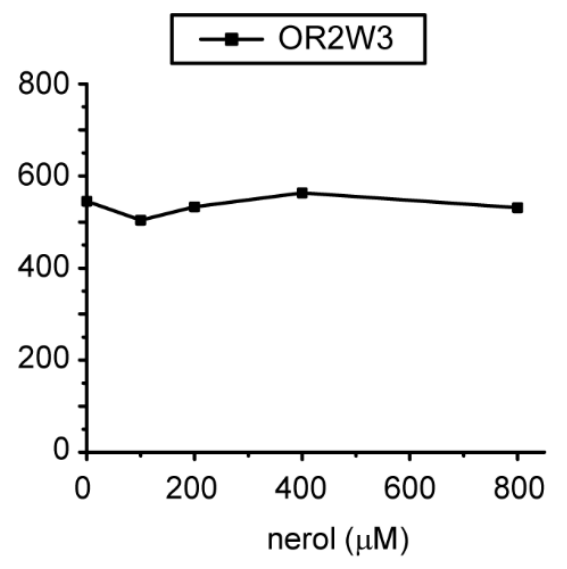

FIGURE S1. Dose response curve of OR2W3 with nerol.
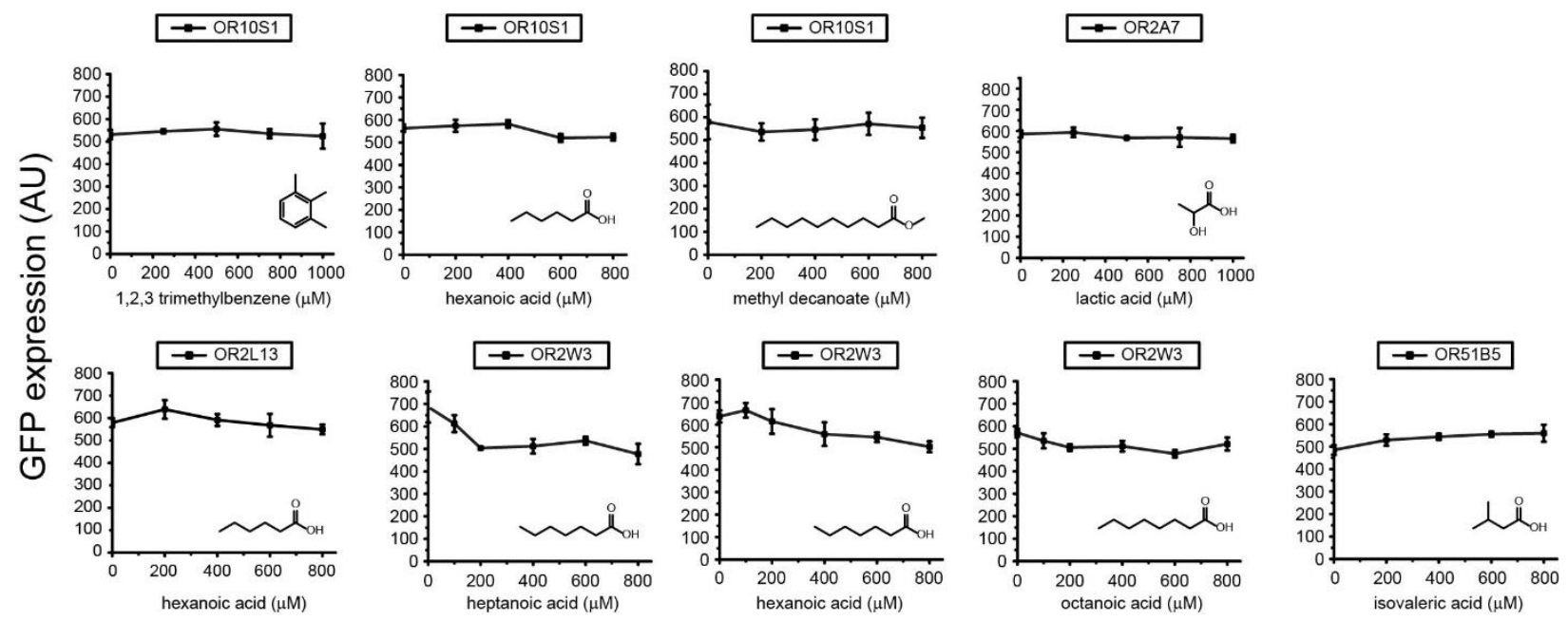

Figure S2. Dose response curves of colon ORs with chemical hits (false positives). Chemicals shown above did not elicit a statistically significant increase in GFP expression. 
A
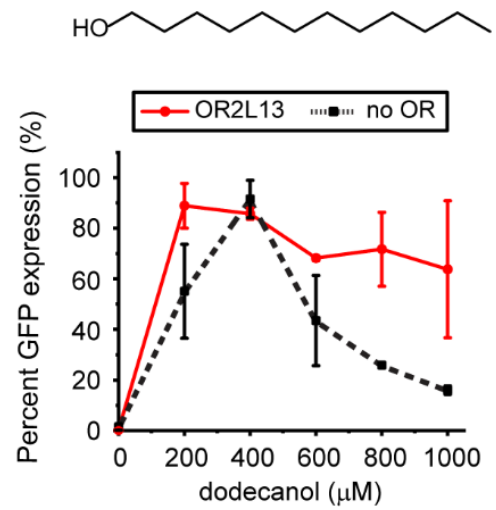

B
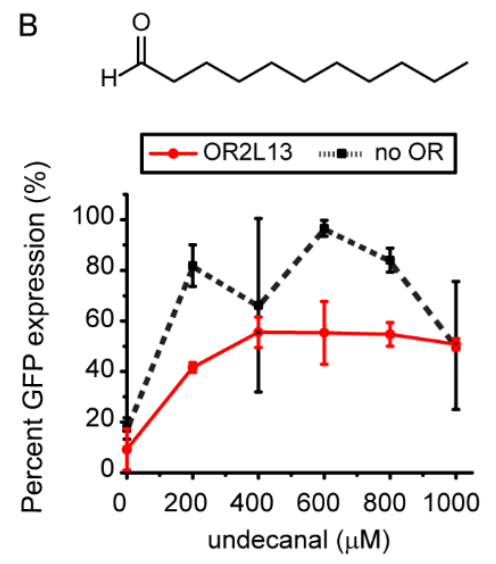

C
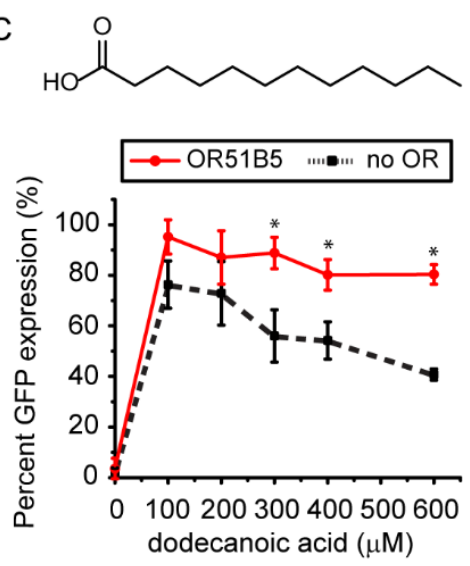

Figure S3. Dose response curves of OR2L13 and OR51B5 with validated chemicals. A. OR2L13 with dodecanol. B. OR2L13 with undecanal. C. OR51B5 with dodecanoic acid.
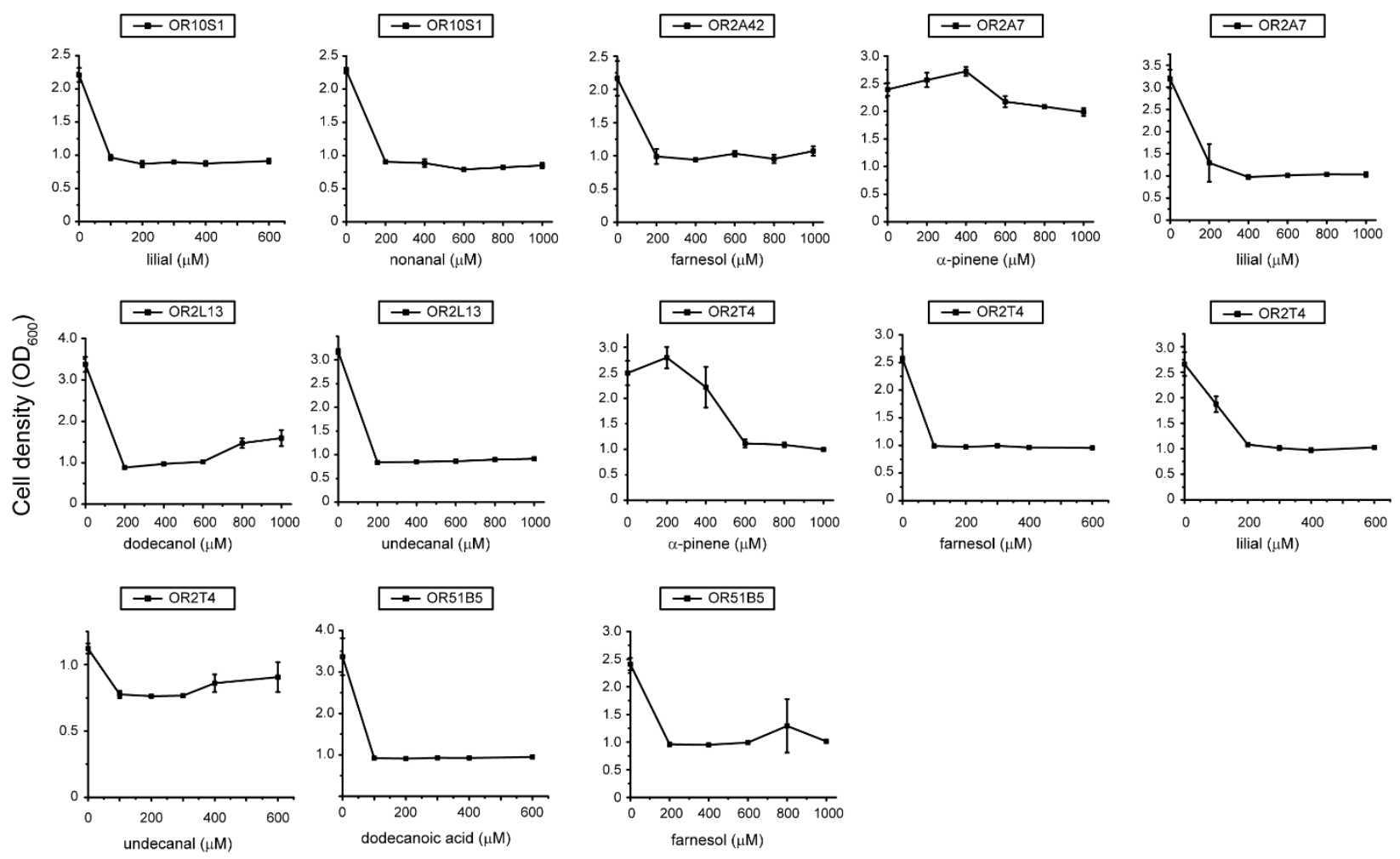

FIGURE S4. Chemical toxicity to yeast-based OR sensor. Yeast cell density was measured after the 4 hour incubation step in the OR chemical sensing protocol. The no chemical data point has $1 \%$ DMSO. While the yeast cells double in the absence of chemical, their cell density is static in the presence of chemical. 


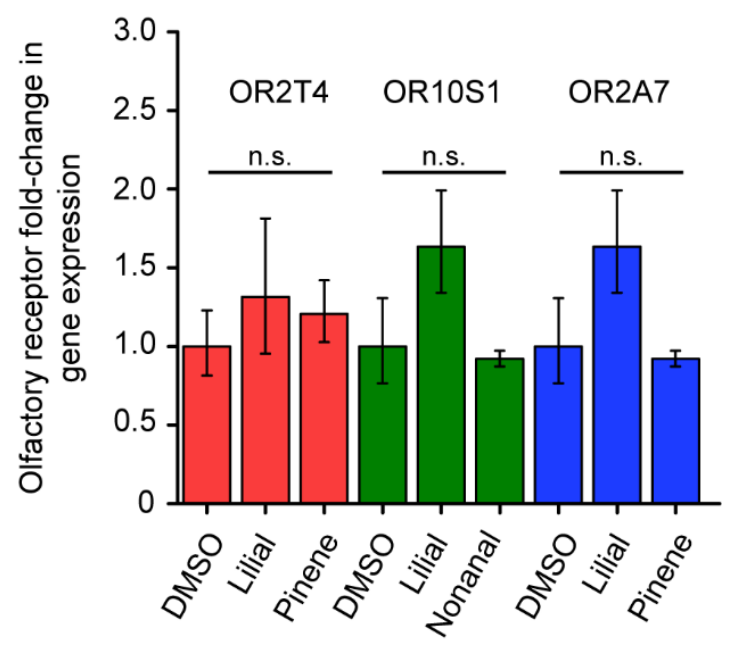

FIGURE S5. Changes in olfactory receptor gene expression levels in the presence of chemicals. Red bars: OR2T4. Green bars: OR10S1.Blue bars: OR2A7. The gene expression experiments were run $600 \mu \mathrm{M}$ lilial and nonanal, and $1000 \mu \mathrm{M}$ pinene. DMSO controls were compared to OR/chemical pairs for OR2T4, OR10S1, and OR2A7 and in all cases P>0.05.

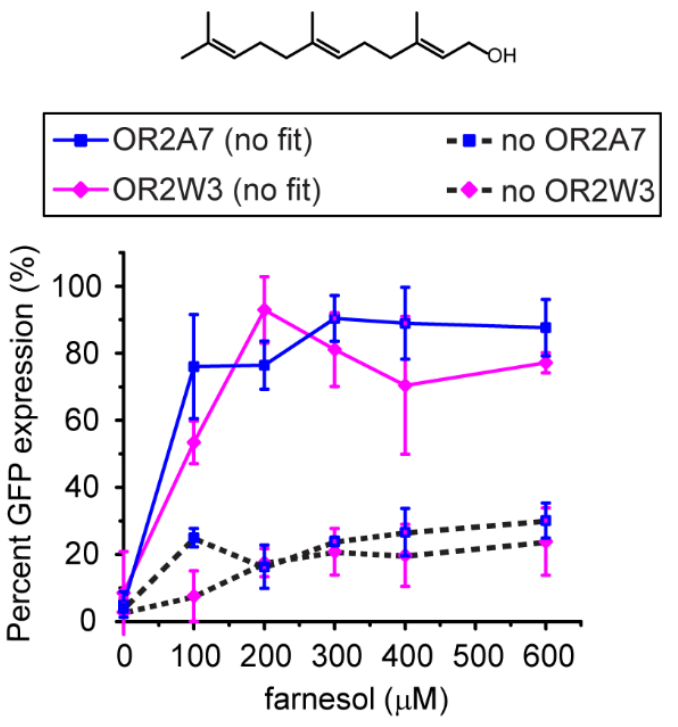

FIGURE S6. Dose response curves of OR2A7 and OR2W3 with farnesol. 


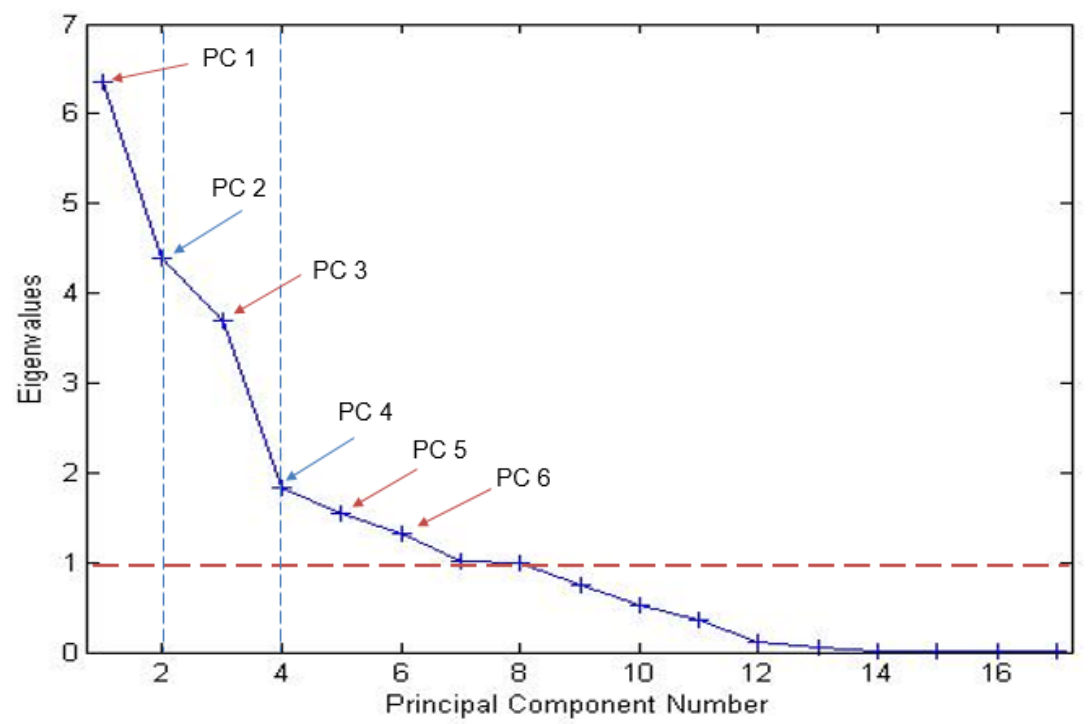

Figure S7. Eigenvalues for principle component determination. Two criteria to be considered in determining number of principle components are: 1) number of PCs with eigenvalues greater than one (red dashes and red arrows), 2) PCs located after bends or knees (blue dashes and blue arrow). Solo analysis resulted in six eigenvalues greater than one, thus six PCs (PCs 1 to 6 ) could be considered for the analysis. Two bends were also observed on the graph at PC2 and PC4, meaning that the PCs after the bends (PC3 and PC5) need to be included in the analysis. These criteria led us to include three PCs (PCs 1-3) in our analysis.

\section{Sequences}

Homo sapiens OR2A42 (UniprotKB - Q8NGT9)

ATGGGTGAAAATCAAACTATGGTTACCGAATTTCTACTACTAGGTTTCCTACTAGGTCCAAG AATCCAAATGCTACTATTCGGTTTGTTCTCCTTGTTCTACATTTTCACCTTGTTGGGTAACG GTGCTATTTTGGGTTTGATTTCCTTGGATTCCAGATTGCATACCCCAATGTATTTCTTCCTAA GTCATTTGGCTGTTGTTGATATTGCTTACACCAGAAACACCGTTCCACAAATGTTGGCTAAC TTGTTGCACCCTGCCAAGCCTATTAGCTTCGCCGGATGCATGACCCAAACCTTCTTATGTTT ATCCTTCGGTCATTCCGAATGTTTGTTGTTGGTTTTGATGTCTTACGATAGATACGTTGCTA TTTGTCATCCATTGAGATACTCCGTTATTATGACTTGGAGAGTTTGTATTACTTTGGCTGTTA CTTCCTGGACTTGTGGTTCCTTGTTGGCTTTGGCTCATGTTGTTTTGATTTTGAGATTGCCA TTCTCCGGCCCACATGAAATTAACCATTTCTTCTGTGAAATTTTGTCCGTTTTGAGATTGGC TTGTGCTGATACTTGGTTGAACCAAGTTGTTATTTTCGCTGCTTGTGTCTTCTTCCTAGTTG GTCCACCATCTTTGGTTTTGGTTTCTTACTCTCATATTTTGGCTGCTATTTTGAGAATACAAT CTGGCGAAGGCAGAAGAAAGGCTTTCTCTACTTGTTCTTCTCATTTGTGTGTTGTTGGTTTG TTCTTCGGTTCTGCTATTATTATGTACATGGCTCCAAAGTCTAGACATCCAGAAGAACAACA AAAGGTTTTCTTCTTGTTCTACTCTTTCTTCAACCCAACTTTGAACCCATTGATTTACTCTTT GAGAAACGGCGAAGTTAAGGGTGCTTTGAGGAGGGCTTTGGGCAAGGAATCTCATTCTTA A

Homo sapiens OR2W3 (UniprotKB - Q7Z3T1)

ATGGATGGTACTAACGGTTCCACCCAAACCCACTTTATTCTATTGGGTTTCTCCGATAGACC ACATTTGGAAAGAATTTTGTTCGTTGTTATTTTGATTGCTTACTTGTTGACCTTGGTTGGTAA CACCACCATTATTTTGGTTTCCAGACTAGACCCACATTTGCATACCCCAATGTACTTCTTCT TGGCTCATTTGTCCTTCTTGGATTTGTCTTTCACCACCTCCTCCATTCCACAATTGTTGTACA 
ACTTGAACGGTTGTGATAAGACCATTTCCTACATGGGTTGTGCTATTCAATTGTTCTTGTTC TTGGGTTTGGGTGGTGTTGAATGTTTGTTGTTGGCTGTTATGGCTTACGATAGATGTGTTG CTATTTGTAAGCCATTGCATTACATGGTTATTATGAACCCAAGATTGTGTAGAGGTTTGGTT TCTGTTACCTGGGGTTGTGGTGTCGCTAACTCTTTGGCTATGTCTCCAGTCACTCTACGTC TACCAAGATGTGGCCATCATGAAGTTGATCATTTCTTGAGAGAAATGCCAGCTTTGATTAGA ATGGCTTGTGTTTCTACTGTTGCTATTGAAGGTACTGTTTTCGTTTTGGCTGTTGGTGTTGT TCTAAGTCCATTGGTTTTCATTTTGTTGTCTTACTCTTACATTGTTAGAGCTGTTCTACAAAT TAGATCTGCTTCTGGTAGACAAAAGGCTTTCGGTACTTGTGGTTCTCATTTGACTGTTGTTT CTTTGTTCTACGGTAACATTATTTACATGTACATGCAACCAGGTGCTTCTTCTTCTCAAGATC AAGGTATGTTTCTAATGCTATTCTACAACATTGTTACTCCATTGTTGAACCCCCTAATCTACA CTCTACGTAACCGTGAAGTCAAGGGTGCTTTGGGTAGATTGTTGTTGGGTAAGAGAGAACT AGGCAAGGAATAA

Homo sapiens OR2T4 (UniprotKB - Q8NH00)

ATGGGTCTGTTCAGACAGAGCAAGCACCCAATGGCTAACATTACCTGGATGGCTAACCATA CCGGTTGGAGCGATTTCATTCTGCTGGGTCTGTTCAGACAATCCAAGCATCCAGCTCTGCT GTGTGTCGTCATTTTCGTTGTTTTCTTGATGGCTTTGTCCGGTAACGCTGTCCTAATCTTGT TGATTCATTGTGATGCTCATTTGCATACCCCAATGTACTTCTTCATTTCCCAATTGTCCTTGA TGGATATGGCTTACATTTCCGTTACCGTTCCAAAGATGTTGTTGGATCAAGTTATGGGTGTT AACAAGATTTCCGCTCCAGAATGTGGTATGCAAATGTTCTTCTACGTTACCTTGGCTGGTTC CGAGTTCTTTCTGTTGGCTACTATGGCTTACGATAGATACGTTGCTATTTGTCATCCATTGA GATACCCAGTTTTGATGAACCATAGAGTTTGTTTGTTCTTGTCCTCCGGTTGTTGGTTCTTG GGTTCCGTCGATGGTTTTACCTTCACCCCAATTACCATGACCTTCCCATTCAGAGGTTCCA GAGAAATTCACCACTTCTTCTGTGAAGTTCCAGCTGTTTTGAACTTGTCCTGTTCCGATACT TCCTTGTACGAAATTTTCATGTACTTGTGTTGTGTTTTGATGTTGTTGATTCCAGTTGTTATT ATTTCTTCTTCTTACTTGTTGATTTTGTTGACTATTCATGGTATGAACTCTGCTGAAGGTAGA AAGAAGGCTTTCGCTACTTGTTCTTCTCATTTGACTGTTGTTATTTTGTTCTACGGTGCTGCT ATTTACACTTACATGTTGCCATCTTCTTACCATACTCCAGAGAAGGATATGATGGTTTCTGTT TTCTACACTATTTTGACTCCAGTTGTTAACCCATTGATTTACTCTTTGCGTAACAAGGATGTT ATGGGTGCTCTAAAGAAGATGTTGACTGTTGAACCAGCCTTCCAAAAGGCAATGGAATAA

Homo sapiens OR51B5 (UniprotKB - Q9H339)

ATGTCCAGCTCCGGCTCCTCCCATCCATTCTTGTTGACCGGCTTCCCAGGTTTGGAAGAAG CTCATCATTGGATTTCCGTTTTCTTCTTGTTCATGTACATTTCCATTTTGTTCGGTAACGGTA CCCTACTACTACTAATTAAGGAAGATCATAACTTGCATGAGCCTATGTATTTCTTCTTGGCT ATGTTGGCTGCTACCGATTTGGGTTTGGCTTTGACCACCATGCCAACCGTTTTGGGTGTTT TGTGGTTGGATCATAGAGAGATTGGTTCTGCCGCCTGTTTCTCCCAAGCCTACTTCATTCAT TCCTTGTCCTTCTTGGAATCCGGCATTTTGTTGGCTATGGCTTACGATCGTTTCATTGCTAT TTGTAACCCATTGAGATACACCTCCGTTCTAACTAATACTCGTGTTGTTAAGATTGGCTTGG GTGTTTTGATGAGAGGTTTCGTTTCCGTTGTTCCACCAATTAGACCATTGTACTTCTTCTTG TACTGTCATTCCCATGTTTTGTCCCATGCTTTCTGTTTGCATCAAGATGTTATTAAGTTGGCT TGTGCTGATACTACTTTCAACAGATTGTACCCAGCTGTTTTGGTTGTTTTCATTTTCGTTTTG GATTACTTGATCATCTTCATTAGTTACGTCCTAATTTTGAAGACTGTTTTGTCCATTGCTTCC AGAGAAGAAAGAGCTAAGGCTTTGATTACTTGTGTTAGTCATATTTGTTGTGTTTTGGTTTT CTACGTTACTGTTATTGGTCTAAGTTTGATTCATCGTTTCGGAAAACAGGTCCCACATATTG TTCATTTGATTATGTCCTACGCTTACTTCCTATTCCCTCCACTAATGAACCCAATTACTTACT CCGTTAAGACCAAGCAAATTCAAAACGCTATTTTGCATTTGTTCACCACCCATAGAATTGGT ACCTAA

Homo sapiens OR2L13 (UniprotKB - Q8N349)

ATGGAGAAGTGGAATCATACATCAAACGACTTCATCCTTCTGGGTCTGTTACCTCCTAATCA GACCGGTATCTTCCTGCTCTGTCTGATCATTCTGATCTTCTTCCTGGCAAGCGTTGGTAATA 
GCGCAATGATTCATCTGATTCACGTTGATCCGCGTCTGCATACCCCGATGTATTTCCTGCT GAGCCAGCTGAGCCTGATGGATCTGATGTATATTAGTACCACCGTTCCGAAGATGGCATAT AATTTCTTAAGTGGTCAGAAGGGCATCAGTTTCTTAGGTTGTGGTGTTCAAAGTTTCTTCTT CTTAACAATGGCCTGTAGTGAAGGTCTGTTACTCACCTCAATGGCCTACGATCGTTATTTAG CAATTTGTCATTCATTATATTATCCTATTAGAATGTCAAAGATGATGTGCGTTAAGATGATTG GTGGTTCTTGGACATTAGGTTCAATTAACAGTTTAGCACATACAGTGTTCGCATTACATATT CCGTATTGTAGAAGTAGAGCAATTGATCATTTCTTCTGTGACGTTCCTGCTATGTTATTATTA GCTTGTACCGATACCTGGGTTTACGAATATATGGTGTTCGTTAGTACCAGTCTGTTCTTGCT GTTCCCATTCATTGGTATTACCAGTAGTTGTGGTCGTGTTCTGTTCGCAGTTTATCACATGC ATAGCAAGGAAGGCAGAAAGAAGGCATTCACTACTATTAGCACCCATCTGACCGTTGTTAT CTTCTATTACGCACCTTTCGTTTACACCTATCTGCGTCCTCGTAACCTGCGTAGTCCGGCA GAAGATAAGATTCTGGCAGTGTTCTACACCATTCTGACCCCGATGCTGAACCCGATTATTTA TAGCCTGCGTAATAAAGAAGTTCTGGGTGCAATGCGTCGTGTGTTCGGTATCTTCAGCTTC CTGAAGGAATAA

Homo sapiens OR10S1 (UniprotKB - Q8NGN2)

ATGACCAGTCGTAGTGTTTGTGAAAAGATGACCATGACCACCGAAAATCCGAATCAGACAG TTGTTAGCCATTTCTTCTTAGAAGGTTTACGTTATACAGCAAAGCATAGTAGTTTATTCTTCT TATTATTCTTATTAATTTACAGTATTACAGTTGCAGGTAACTTATTAATTTTATTAACCGTTGG TAGTGATAGCCATTTAAGTTTACCGATGTATCATTTCTTAGGTCATTTAAGTTTCTTAGACGC CTGTTTAAGTACCGTTACAGTTCCGAAGGTTATGGCAGGTTTATTAACCTTAGACGGTAAAG TTATTAGTTTCGAAGGTTGTGCAGTTCAGTTATATTGTTTCCACTTCTTAGCAAGTACAGAGT GTTTCTTATATACAGTTATGGCATACGATCGTTATTTAGCAATCTGTCAGCCGTTACATTATC CGGTTGCCATGAATCGTCGTATGTGTGCCGAAATGGCAGGTATTACCTGGGCAATTGGTG CAACCCACGCAGCAATTCATACCAGCCTGACCTTTCGTCTGCTGTATTGTGGTCCGTGTCA TATTGCATATTTCTTCTGTGATATTCCGCCGGTTCTGAAACTGGCCTGTACCGATACCACCA TTAACGAACTGGTTATGTTAGCAAGTATTGGTATTGTTGCAGCAGGTTGTCTGATTCTGATT GTTATTAGTTATATTTTCATTGTTGCAGCAGTTTTACGTATTCGTACAGCACAGGGTCGTCA GCGTGCATTCAGTCCGTGTACAGCACAATTAACAGGTGTTTTATTATATTACGTTCCGCCG GTTTGCATTTATTTACAACCGCGTAGCAGTGAAGCAGGTGCCGGTGCACCGGCAGTTTTCT ATACCATTGTTACACCGATGCTGAATCCGTTTATTTATACATTACGTAATAAGGAGGTTAAA CACGCATTACAACGTTTACTGTGTAGTAGTTTTCGTGAAAGCACCGCAGGTAGCCCGCCGC CGTAA

Homo sapiens OR2A7 (UniprotKB - Q96R45)

ATGGGTGACAACATTACCAGCATTACCGAATTTCTGCTGCTGGGTTTTCCTGTTGGTCCAC GCATCCAGATGCTGCTGTTTGGTCTGTTCAGCCTGTTCTACGTATTTACACTGCTGGGTAA CGGTACCATTTTAGGTTTAATTAGCTTAGATAGCCGTTTACACGCACCTATGTACTTCTTCTT AAGCCATTTAGCAGTTGTTGATATTGCATACGCTTGCAACACAGTTCCTCGTATGTTGGTTA ACTTATTACATCCGGCTAAACCTATTAGCTTCGCAGGTCGTATGATGCAAACCTTCTTATTT AGCACCTTCGCAGTTACCGAGTGTTTATTATTAGTTGTTATGAGCTACGATTTATACGTTGC AATTTGCCATCCATTACGTTACTTAGCAATTATGACCTGGCGTGTTTGTATTACCTTAGCAG TTACAAGCTGGACAACAGGTGTTCTGTTAAGTCTGATTCACTTAGTTTTATTATTACCTTTAC CTTTCTGTCGTCCTCAAAAGATTTATCATTTCTTCTGTGAAATTTTAGCAGTTTTAAAGTTAG CCTGTGCAGACACACACATTAACGAAAACATGGTTTTAGCAGGTGCAATTAGCGGTTTAGT TGGTCCTTTAAGTACCATTGTTGTTAGCTACATGTGTATTCTGTGTGCAATTTTACAAATTCA AAGCCGTGAAGTTCAGCGTAAGGCATTCTGCACCTGTTTCAGCCACCTGTGTGTTATTGGT TTATTCTACGGTACAGCAATTATTATGTACGTTGGTCCGCGTTACGGTAACCCTAAGGAACA AAAGAAGTACTTATTATTATTCCATAGCCTGTTTAACCCGATGCTGAACCCGCTGATTTGTA GCCTGCGTAACAGCGAAGTTAAGAACACCCTGAAGCGCGTTCTGGGTGTTGAACGTGCAC TGTAA 


\section{REFERENCES}

Mukherjee, K., Bhattacharyya, S. \& Peralta-Yahya, P. (2015) GPCR-Based Chemical Biosensors for Medium-Chain Fatty Acids. ACS Synthetic Biology 4, 1261-1269.

Livak, K. J. \& Schmittgen, T. D. (2001) Analysis of relative gene expression data using real-time quantitative PCR and the 2(-Delta Delta C(T)) Method. Methods 25, 402-408. 